\title{
Uma proposta de instrumento de avaliação pedagógica da preceptoria para residências em Medicina de Família e Comunidade
}

\author{
A proposal for a pedagogical assessment instrument for preceptorship for residences in Family Practice \\ Una propuesta para un instrumento de evaluación pedagógica de la preceptoría para residencias \\ en Medicina Familiar y Comunitaria
}

\author{
Pollyane Rodrigues Miranda ${ }^{\oplus}$, Valéria Ferreira Romano ${ }^{2} \odot$ \\ ${ }^{1}$ Unidade de Saúde da Família Barra do Jucu, Secretaria Municipal de Vila Velha, Vila Velha - ES, Brasil \\ 2 Universidade Federal do Rio de Janeiro, PAPS, Rio de Janeiro - RJ,Brasil
}

\section{Resumo}

Introdução: Desde a Conferência de Alma-Ata, a importância do cuidado centrado na Atenção Primária à Saúde (APS) foi consolidada como objetivo mundial. No Brasil, a Estratégia Saúde da Família (ESF), composta por equipes multiprofissionais, visa à reorganização da APS, sendo o especialista em Medicina de Família e Comunidade (MFC) o médico indicado para atuação neste campo. A residência em MFC é considerada o padrão ouro da formação desse profissional, porém, para expansão da residência é necessário expansão da preceptoria. Preceptores são profissionais que utilizam seu conhecimento e sua experiência profissional para atuação docente junto aos residentes. No município do Rio de Janeiro houve expansão da APS, com aumento da cobertura ESF de $3,5 \%$ para $69,43 \%$ em 2018 , o que trouxe a necessidade de investimento para expansão da residência, e, desta forma, demandou mais preceptores. No entanto, não pôde ser realizada uma capacitação completa em tempo hábil, sendo desejável que os preceptores continuassem sua qualificação concomitante à preceptoria. Dentre as atividades do preceptor, destacam-se os atributos pedagógicos, relacionados à responsabilidade docente no processo ensino-aprendizagem do residente. Objetivo: Apresentar uma proposta de instrumento para avaliação dos atributos pedagógicos da preceptoria das residências em Medicina de Família e Comunidade. Métodos: Para elaboração da proposta de instrumento para avaliação dos atributos pedagógicos foram realizadas entrevistas compreensivas, com preceptores em Medicina de Família e Comunidade da Universidade do Estado do Rio de Janeiro, segundo um roteiro semiestruturado. Resultados: As entrevistas geraram espaços de reflexão sobre as práticas pedagógicas dos preceptores, motivando-os a construírem novos planos pedagógicos e a repensarem as potencialidades e os obstáculos enfrentados no trabalho pedagógico da preceptoria em MFC. Conclusão: A proposta de um instrumento para avaliação pedagógica se apresentou como formativa para a preceptoria em MFC no âmbito da APS do município do Rio de Janeiro, fazendo supor que produziu ressignificação, precursor de motivação e esperança.

Palavras-chave: Educação em Saúde; Preceptoria; Avaliação Educacional; Medicina de Família e Comunidade.

Como citar: Miranda PR, Romano VF. Uma proposta de instrumento de avaliação pedagógica da preceptoria para residências em Medicina de Família e Comunidade. Rev Bras Med Fam Comunidade. 2021;16(43):2680. https://doi.org/10.5712/rbmfc16(43)2680

\author{
Autor correspondente: \\ Pollyane Rodrigues Miranda. \\ E-mail: pollyanermiranda@gmail.com \\ Fonte de financiamento: \\ não se aplica. \\ Parecer CEP: \\ UFRJ CAAE 14846519.2.0000.5238 / \\ SMSRJ CAAE 14846519.2.3001.5279 \\ Procedência: \\ não encomendado. \\ Avaliação por pares: \\ externa \\ Recebido em: 18/08/2020. \\ Aprovado em: 23/12/2020. \\ Editor Associado: \\ Luciana Tricai Cavalini
}




\begin{abstract}
Introduction: Since Alma-Ata Conference, the importance of Primary Health Care (PHC) has been consolidated as a global goal. In Brazil, the Family Health Strategy (FHS), made up of multi-professional teams, aims at the reorganization of APS, where the physician indicated for performance in this field is the expert in Family Practice (FP). The FP residency is considered the gold pattern for specialization of this professional, however, for expansion of residency, it is necessary to expand the preceptorship. Preceptors are professionals who use their knowledge and their professional experience for teaching practice to residents. In the city of Rio de Janeiro, there was an expansion of the PHC, with an increasing in FHS coverage from $3.5 \%$ to $69.21 \%$ in 2018 , which brought the need for investment to expand the FP residency, and thus demanded more preceptors. However, the full training could not be completed in time, but the wish was that preceptors continue their qualification concomitantly with preceptorship. Among the activities of the preceptor, the pedagogical attributes related to the responsibility in the teaching-learning process of the resident stand out. Objective: To present a proposal for an instrument to assess the pedagogical attributes of the preceptorship of residences in FP. Methods: Comprehensive interviews were carried out to develop the proposed instrument for the assessment of pedagogical attributes, with preceptors in FP from the Universidade do Estado do Rio de Janeiro, according to a semi-structured script. Results: The interviews generated spaces for reflection on the pedagogical practices of the preceptors, motivating them to build new pedagogical plans and to rethink the potentialities and obstacles faced in the pedagogical work of preceptorship in FP. Conclusion: The proposal of an instrument for pedagogical assessment was presented as formative for the preceptorship in FP within PHC in the city of Rio de Janeiro, suggesting that it produced resignification, a precursor of motivation and hope.
\end{abstract}

Keywords: Health Education; Preceptorship; Educational Measurement; Family Practice.

\title{
Resumen
}

Introducción: Desde la Conferencia de Alma-Ata, la importancia de la atención centrada en la Atención Primaria de Salud (APS) se ha consolidado como un objetivo global. En Brasil, la Estrategia de Salud Familiar (ESF), compuesta por equipos multiprofesionales, tiene como objetivo reorganizar la APS, siendo el médico especialista en Medicina Familiar y Comunitaria (MFC) el indicado para trabajar en este campo. La residencia en MFC se considera el estándar de oro de la formación de este profesional, sin embargo, para ampliar la residencia, es necesario ampliar la preceptoría. Los preceptores son profesionales que utilizan sus conocimientos y experiencia profesional para enseñar la practica a los residentes. En el municipio de Río de Janeiro hubo una expansión de APS, con un aumento en la cobertura de la ESF de 3.5\% a 69.43\% en 2018 , lo que trajo la necesidad de inversión para expandir la residencia y, por lo tanto, exigió más preceptores. Sin embargo, una calificación completa no podría llevarse a cabo de manera oportuna, siendo deseable que los preceptores continúen su calificación concomitante con la preceptoría. Entre las actividades del preceptor, se destacan los atributos pedagógicos, relacionados con la responsabilidad docente en el proceso de enseñanza-aprendizaje del residente. Objetivos: Presentar una propuesta de instrumento para evaluar los atributos pedagógicos de la preceptoría de residencias en MFC. Métodos: Se realizaron entrevistas comprensivas para desarrollar el instrumento propuesto para la evaluación de los atributos pedagógicos, con preceptores en MFC de la Universidade do Estado do Rio de Janeiro, según un guion semiestructurado. Resultados: Las entrevistas generaron espacios de reflexión sobre las prácticas pedagógicas de los preceptores, motivándolos a construir nuevos planes pedagógicos y a repensar las potencialidades y obstáculos enfrentados en el trabajo pedagógico de la preceptoría en la MFC. Conclusión: La propuesta de un instrumento para la evaluación pedagógica se presentó como formativa para la preceptoría en MFC en el ámbito de la APS de la ciudad de Río de Janeiro, lo que sugiere que produjo resignificación, un precursor de la motivación y la esperanza.

Palabras clave: Educación en Salud; Preceptoría; Evaluación Educacional; Medicina Familiar y Comunitaria.

\section{INTRODUÇÃO}

O conceito de saúde e a visão do processo saúde-doença passaram por renovações inspiradas, principalmente, pelas discussões da Conferência de Alma-Ata - primeira conferência internacional sobre cuidados primários em saúde, organizada pela ONU e UNICEF. A Declaração produzida nesse evento consolidou a relevância de um modelo de cuidados centrado e fundamentado na Atenção Primária à Saúde (APS) como grande objetivo político e social a ser atingido pelos países de todo o mundo. ${ }^{1} \mathrm{Um}$ sistema de saúde baseado na APS é aquele instituído por um conjunto central de componentes funcionais e estruturais que garantem a cobertura e o acesso universais a serviços apropriados à população e que melhoram a equidade. ${ }^{2}$

No Brasil, a Estratégia Saúde da Família (ESF) ocupa o espaço de reorganização da APS e representa uma estratégia para sua expansão, qualificação e consolidação. Tem a capacidade de reorientar o processo de trabalho com maior valorização do Sistema Único de Saúde (SUS) em seus princípios, diretrizes e fundamentos, de aumentar a resolutividade e obter impacto na situação de saúde individuais e comunitárias, propiciando significativa relação custo-efetividade. ${ }^{2}$ 
Em relação ao profissional médico na ESF, a especialidade orientada notoriamente para os cuidados primários em saúde é a Medicina de Família e Comunidade (MFC), pois estes médicos realizam atenção integral, ampla e em todos os níveis de atenção à saúde ${ }^{1}$; ou seja, a especialização em MFC, promove maior capacidade de produzir cuidados abrangentes e continuados a todos os sujeitos, independente de idade, sexo ou condição de saúde.

A residência médica é considerada o padrão ouro para especialização em determinada área. Desta maneira, a Comissão Nacional de Residência Médica é responsável por determinar os requisitos mínimos dos programas de residência médica em MFC, bem como os requisitos de todas as residências médicas. ${ }^{3}$ No Rio de Janeiro, o processo de expansão da APS de 3,5\% em 2009 para 69,43\% em $2018^{4}$ trouxe a necessidade de investimento municipal para a formação de novos profissionais médicos de família e comunidade através da expansão das vagas de residência médica já existentes - ligadas à Universidade Federal do Rio de Janeiro (UFRJ), à Escola Nacional de Saúde Pública (ENSP) e à Universidade do Estado do Rio de Janeiro (UERJ) - e na criação de um novo programa de residência em MFC, ligado diretamente a Secretaria Municipal de Saúde do Rio de Janeiro (SMS-RJ). ${ }^{5}$ Um contexto de crise na atenção primária à saúde do sistema de saúde carioca a partir de $2018^{6}$ provocou queda dessa cobertura para $50,27 \%$ em março de $2020,{ }^{4}$ porém o município ainda permanece com um dos maiores programas de residência em MFC do Brasil.

Certamente, para uma expansão da APS com médicos de família e comunidade qualificados, é necessária também a expansão do número de preceptores no âmbito da APS. Preceptores são profissionais do ofício que mobilizam seu domínio de saberes e sua experiência na área em que trabalham para atuação docente, neste caso com os residentes, articulando aprendizagem e práticas cuidadoras. ${ }^{7}$ Nos Programas de Residência em Medicina de Família e Comunidade (PRMFC), o preceptor conduz o percurso do desenvolvimento profissional do médico residente tanto para o manejo clínico e abordagem individual, familiar e comunitária, quanto para a gestão e organização dos serviços de saúde. ${ }^{8}$ No município do Rio de Janeiro, os residentes atuam como médicos responsáveis pelas equipes de Saúde da Família sob orientação da preceptoria durante os dois anos da residência como estágio longitudinal no âmbito da APS. Na UERJ ainda há o terceiro ano, o R3 com foco em preceptoria e gestão, chamado de residência em Administração em Saúde, em que os residentes, ainda inseridos nas equipes de Saúde da Família, agregam lugar de referência profissional para os residentes em MFC, como copreceptores.

Como já citado, o rápido crescimento carioca da residência em MFC resultou em uma demanda por um grande número de preceptores com requisitos que atendessem ao processo de expansão, de forma que não pôde ser realizada uma capacitação em preceptoria de maneira mais plena, em tempo hábil. Uma concomitância tornou-se imprescindível: os profissionais continuariam seus processos de formação e qualificação de competências, através do apoio das instituições às quais estavam vinculados, em sincronia ao exercício da própria preceptoria.

Seguramente, dentre as atribuições da preceptoria, destaca-se a responsabilidade no processo de ensino-aprendizagem do residente - relacionada ao domínio de atributos pedagógicos, a competência técnica como especialista e a responsabilidade pela formação moral e ética do residente. ${ }^{9} \mathrm{O}$ domínio de atributos pedagógicos está relacionado ao papel do preceptor como educador, sendo importante para oportunizar ao residente ambientes de construção e reconstrução de conhecimentos, habilidades e atitudes. ${ }^{10}$ 
Nesse contexto, as metodologias ativas ganham destaque, pois proporcionam a construção do aprendizado a partir da vivência de situações reais dos residentes e têm o intuito de torná-los corresponsáveis pela construção do próprio processo de aprendizado. O processo de ensino-aprendizagem passa a acontecer em uma relação de troca enriquecedora entre educador, educando e conteúdo. ${ }^{11}$ Este contexto de ensino-aprendizagem condiz com aquele trabalhado na educação problematizadora, baseada no referencial teórico de Freire, ${ }^{12}$ em que os educandos vão construindo sua compreensão de mundo através de suas relações com ele, de forma que a realidade não se mostra mais imóvel, mas em processo, em transformação. ${ }^{12}$ Em associação à educação problematizadora, hooks ${ }^{13}$ defende o ideal de uma educação libertadora, em que o educador participa do crescimento intelectual e espiritual dos alunos trabalhando em conjunto com eles, respeitando sua realidade social e experiência multicultural nesse processo pedagógico.

Dentro de seu modelo educacional, Perrenoud ${ }^{14}$ dispõe o ato de relacionar o currículo à prática real do aluno como uma das habilidades necessárias ao educador, o que envolve o conhecimento da comunidade em que estão inseridos. Desta forma, a escola, como um espaço de ensino-aprendizagem que dialoga com o contexto social em que está inserido, deve se renovar e representar um espaço democrático professoraluno-comunidade ${ }^{14}$ consonante aos ideais da educação problematizadora e libertadora discutidos acima. Acreditamos na relevância dos PRMFC fazerem eco à estas características, já que estão envolvidos com a formação de profissionais que se autorizam a compreender e trabalhar o processo saúde-doença nas esferas individual, familiar e comunitária de forma crítica, segundo a realidade em que estão inseridos; tornando possível uma correlação pedagógica dos PRMFC com o modelo escolar descrito pelo autor.

Em Dez novas competências para ensinar, ${ }^{15}$ Perrenoud caracteriza as competências necessárias aos professores para ensinar, com base em sua teoria pedagógica. Ele defende que, antes de inserir-se como educador no processo de ensino-aprendizagem, o professor deve reconhecer suas próprias competências individuais, mapeando possibilidades e limites. Ao identificar esses limites, deve procurar desenvolver tal competência que não possui e adequar as possuídas aos usos a que se destinam, mobilizando sua capacidade de avaliação. Transportando-se esse processo para o desenvolvimento de atributos pedagógicos na preceptoria dos PRMFC, uma avaliação de caráter formativo permite que os preceptores reflitam sobre suas práticas docentes e se sensibilizem quanto às potencialidades e aos obstáculos em seus contextos de trabalho com os residentes. A partir daí, os preceptores têm a possibilidade de construir novos planos para atuação docente e desenvolver os atributos pedagógicos avaliados.

Neste processo, a avaliação tem papel importante de informação e orientação para melhoria de competências, conforme o modelo em que avaliação e planejamento caminham juntos, defendido por Depresbiteris. ${ }^{16,17}$

Relacionando a avaliação com a qualidade pedagógica da preceptoria nos PRMFC, entendemos a consonância de vários fatores envolvidos para que ela aconteça com relevância - contexto, estrutura, recursos, fortalezas, fragilidades, dentre outros - assumindo, assim, a complexidade que é avaliar, especialmente na preceptoria dos PRMFC, que possuem particularidades nas atividades desenvolvidas junto aos residentes, dentro das Unidades Básicas de Saúde.

O objetivo deste artigo é, portanto, apresentar a construção de um instrumento de avaliação formativa de atributos pedagógicos dos preceptores em MFC, na perspectiva de que seja uma ferramenta que possa auxiliar o desenvolvimento pedagógico como parte do crescimento profissional focado na melhoria do desempenho no exercício da preceptoria no âmbito da APS do município do Rio de Janeiro. 


\section{MÉTODOS}

Este artigo foi gerado a partir de um estudo que realizou uma pesquisa exploratório-descritiva ${ }^{18}$ sobre os atributos pedagógicos da preceptoria em MFC relacionados às Dez novas competências para ensinar de Perrenoud.$^{15}$ A pesquisa inicial foi aplicada no PRMFC da UERJ, onde foi possível construir uma ferramenta para avaliação formativa.

O campo para a pesquisa foram as unidades de saúde pertencentes ao PRMFC UERJ localizadas em uma área programática do município do Rio de Janeiro. Os indivíduos incluídos foram os preceptores médicos do PRMFC UERJ atuantes nessas unidades durante o segundo semestre de 2019. Foram excluídos os preceptores que não estavam atuando nessas unidades no período da pesquisa, além dos que não puderam ou não quiseram participar. Foram entrevistados 13 do total de 15 preceptores que atuavam na abrangência do universo da pesquisa. Cada entrevista durou cerca de 50 minutos a uma hora e meia, em média.

Para coleta de dados e subsequente análise e composição do instrumento de avaliação proposto, foi realizado um método específico de entrevista: a entrevista compreensiva. ${ }^{19}$

A entrevista compreensiva é um método que utiliza técnicas de pesquisa qualitativa e empírica, sobretudo as etnológicas de trabalho íntimo com informantes, porém os dados recolhidos estão concentrados através da palavra recolhida pelo gravador. O entrevistador está ativamente envolvido nas questões justamente para provocar o envolvimento do entrevistado. ${ }^{19}$

Como guia para entrevista foi utilizado um roteiro contendo perguntas de acordo com atributos pedagógicos da preceptoria em MFC considerados a partir da correlação às Dez novas competências para ensinar de Perrenoud. ${ }^{15} \mathrm{O}$ instrumento idealizado foi um roteiro de entrevista semiestruturada, que combinou perguntas fechadas e abertas, possibilitando que o entrevistado desenvolvesse o discurso sobre o tema, e não apenas se prendesse à formulação específica. ${ }^{18,20}$

O roteiro da entrevista continha perguntas sobre informações básicas de formação e experiência profissional para se compreender o perfil dos preceptores entrevistados, e, ao final, foi adicionado um tópico de encerramento para problematização acerca dos sentimentos dos preceptores ao refletirem sobre suas práticas pedagógicas.

Em conformidade com o método da entrevista compreensiva, a interpretação do material não foi afastada no processo de análise de dados, mas sim pretendida por constituir elemento decisivo. ${ }^{19} \mathrm{O}$ procedimento de análise realizado foi a análise de conteúdo, buscando-se a compreensão dos significados no contexto da fala através de indicadores que permitiam a inferência de conhecimentos em relação às condições de produção das mensagens. ${ }^{21}$

O projeto de pesquisa foi submetido para apreciação pelo Comitê de Ética em Pesquisa da Escola de Enfermagem Anna Nery - Hospital Escola São Francisco de Assis da Universidade Federal do Rio de Janeiro com Certificado de Apresentação para Apreciação Ética de número 14846519.2.0000.5238, sendo autorizada a coleta de dados. A SMS-RJ, instituição coparticipante da pesquisa, também concordou com os termos do projeto da pesquisa - Certificado de Apresentação para Apreciação Ética de número 14846519.2.3001.5279.

\section{RESULTADOS}

A partir do roteiro utilizado em campo e da análise das entrevistas com os preceptores atuantes na APS, foi construído o instrumento proposto para avaliação formativa dos atributos pedagógicos da preceptoria em MFC, apresentado a seguir (Quadro 1). 


\section{Quadro 1. Instrumento de Avaliação Pedagógica da Preceptoria de Medicina de Família e Comunidade}

Este instrumento representa a proposta de uma ferramenta de avaliação formativa dos atributos pedagógicos do seu trabalho realizado na preceptoria em Medicina de Família e Comunidade (MFC). Reflita e discuta em pares ou em um pequeno grupo de preceptores - de sua unidade, por exemplo - sobre os atributos pedagógicos a seguir, considerando as potencialidades e os obstáculos existentes no contexto em que você se encontra inserido(a) como preceptor(a) dos residentes de um Programa de Residência em Medicina de Família e Comunidade (PRMFC) no âmbito da Atenção Primária à Saúde (APS).

É importante que haja um ambiente acolhedor, em que você se sinta confortável para discutir, avaliar seu desempenho em relação a esses atributos pedagógicos junto a(os) seu(s) colega(s) preceptor(es) e para construir planos de desenvolvimento profissional individual e em conjunto - ao final da realização desse instrumento. Reservem cerca de uma hora para a realização desta atividade.

\section{CERNE - Atributos Pedagógicos intrínsecos à relação preceptor-residente}

1. Como estão organizados os processos de aprendizagem no seu trabalho com os residentes, incluindo o planejamento dos objetivos, as metodologias utilizadas e a avaliação progressiva? De que forma sua visão longitudinal do programa de ensino contribui para estratégias de ensino-aprendizagem? Além disso, você participa de práticas de pesquisa junto aos residentes como outra forma de envolvimento em projetos de conhecimento?

2. Quais os mecanismos utilizados por você para interação social e heterogeneidade entre os residentes a fim de promover situações de aprendizagem enriquecedoras, visando ao desenvolvimento profissional deles? Que dispositivos, além da figura do preceptor, são utilizados nesse processo interativo?

3. Como você atua para envolvimento dos residentes na aprendizagem dentro do âmbito da APS no sentido de motivá-los e de proporcionar espaços a eles dentro do programa para que construam atividades próprias pertinentes ao desenvolvimento profissional em MFC? Existe algum contrato de convivência para a relação preceptor-residente? Em caso afirmativo, qual foi a participação dos residentes na elaboração desse contrato? E em relação à construção de projetos pessoais dos residentes para além do PRMFC, de que forma você se vê implicado?

ENTORNO - Atributos Pedagógicos que circundam e permeiam a relação preceptor-residente

4. Como você atua para promover o trabalho em equipe entre seus residentes e outros profissionais do ambiente de trabalho na unidade de saúde? Também considerando seu grupo de preceptores local como equipe pedagógica, qual a sua percepção sobre o relacionamento entre vocês tendo em vista aspectos como comunicação, divisão de tarefas e administração de conflitos no dia a dia do exercício da preceptoria?

5. Quais são as relações existentes entre você e a gestão da unidade de saúde, e entre você e a coordenação da residência, no sentido de trabalharem em conjunto para o planejamento das atividades formativas envolvendo os residentes, pacientes e outros trabalhadores da unidade de saúde? De que forma seu grupo de preceptores está organizado como grupo pedagógico para expressar o trabalho realizado por vocês e também para formular projetos para o PRMFC?

6. Quais movimentos e atividades são realizadas por você, preceptor, para envolver e integrar a comunidade adscrita à sua unidade de saúde ao projeto pedagógico da residência? Como lida com os questionamentos que possam surgir acerca do PRMFC, da unidade e de você (preceptor)?

7. De que forma você utiliza novas tecnologias - internet, aplicativos, e-books, entre outros - como potencialidades didáticas no processo de aprendizagem dos residentes? Como é realizada a mediação com eles para o uso racional dessas ferramentas em relação à escolha de referências confiáveis, compatíveis, e à individualização dos pacientes?

8. Relembre uma situação em que você atuou como mediador de algum dilema ético - como em conflitos envolvendo residentes entre si, pacientes e residentes, relacionamento dentro da equipe ou, inclusive, entre preceptor e residente. Como essas situações podem ser utilizadas para construção de valores dos residentes de forma que entendam a importância do aprendizado adquirido nessas mediações para a formação profissional deles? E você, preceptor, como enfrenta o dilema de mediar o desenvolvimento dos residentes em um ambiente mais protegido, enquanto a realidade do trabalho em MFC é mais dura, com mais problemas?

9. Considerando a importância de sua autoformação contínua, como você atua para buscar a diversificação e a atualização de suas ferramentas clínicas e pedagógicas utilizadas no trabalho de ensino-aprendizagem com os residentes? Qual apoio institucional do PRMFC ou externo a ele para sua formação profissional contínua? O envolvimento em atividades de coordenação do PRMFC ou na formação de colegas preceptores representam outros caminhos formativos. Você se percebe implicado em algum deles? Como?

\section{ENCERRAMENTO - Planos para desenvolvimento dos Atributos Pedagógicos}

10. A partir da discussão e avaliação com seu(s) par(es) sobre seu desempenho em relação a esses atributos pedagógicos, construa planos para seu desenvolvimento profissional, individualmente e em conjunto, considerando suas potencialidades e obstáculos individuais, do seu grupo de preceptoria local e/ou de seu PRMFC. Ao final, compartilhe com seu(s) par(es) como você se sentiu durante essa atividade em relação à reflexão de suas práticas pedagógicas como preceptor.

\section{DISCUSSÃO}

O instrumento proposto expressa os atributos pedagógicos da preceptoria em MFC categorizados em dois grandes grupos de questões - o Cerne e o Entorno. O Cerne traz os atributos pedagógicos relacionados 
mais intimamente à relação direta preceptor-residente - os processos de aprendizagem e progressão, os mecanismos de interação social e o envolvimento dos residentes nas atividades, os quais se relacionam às quatro primeiras competências de Perrenoud. ${ }^{15} \mathrm{O}$ Entorno contém os atributos correspondentes a aspectos que circundam e permeiam essa relação preceptor-residente - o trabalho em equipe, a relação do preceptor com a gestão e a coordenação, o papel exercido pela comunidade, o uso de tecnologias, os dilemas éticos no dia a dia e a administração da formação contínua pelo próprio preceptor. Esses atributos correspondem às demais seis competências de Perrenoud, ${ }^{15}$ respectivamente.

As entrevistas se mostraram transformadoras no modo em que permitiram aos preceptores espaços de reflexão sobre suas práticas pedagógicas e proporcionaram a consciência de que muitas práticas realizadas no exercício da preceptoria representavam a utilização de atributos pedagógicos. A partir desses processos, foram provocadas inquietações, com novos pensares e questionamentos a partir dessas reflexões realizadas pelos preceptores, que contribuíram para formulação de novos planos pedagógicos discutidos durante as entrevistas, considerando as potencialidades e obstáculos vivenciados pelos preceptores em questão, tanto individualmente quanto em relação ao grupo de preceptores a que pertenciam.

Uma preocupação exposta pelos entrevistados foi sobre como a avaliação seria aplicada devido ao receio de que surgissem situações constrangedoras nesse processo. Como a entrevista compreensiva proporciona a aproximação entre entrevistado e entrevistador, transportou-se para aplicação do instrumento a sugestão de que a avaliação formativa fosse realizada entre pares ou em pequenos grupos de preceptores que trabalhem em conjunto, almejando-se um ambiente mais acolhedor. Desse modo, espera-se que eles sejam capazes de discutir abertamente, avaliar seu desempenho em relação a esses atributos pedagógicos e, em seguida, construir planos de desenvolvimento para os profissionais envolvidos na execução dessa atividade avaliativa.

É importante situar em que contexto se situavam esses preceptores e todos os trabalhadores da saúde pública carioca no período das entrevistas. Preceptores, residentes e todos os trabalhadores da saúde do Rio de Janeiro constituíam um cenário de resistência à política de corte orçamentário e redução da cobertura da ESF, ${ }^{6}$ medidas que refletiam uma cadeia de políticas de desconstrução e enfraquecimento do SUS nos âmbitos locais e em escala federal nos últimos anos. ${ }^{22}$ Destaca-se a presença do termo "desmonte" em todas as entrevistas, que foi utilizado para descrever a situação de desestruturação da APS, e o manifesto do sentimento de desgaste no processo de envolvimento dos residentes nas atividades de trabalho em meio a essa situação crítica.

\section{CONCLUSÃO}

O preceptor representa um profissional de referência em sua área profissional que realiza atuação docente com os residentes. Como educador, possui papel fundamental na mediação do processo ensino-aprendizagem com seus residentes em ambientes de construção e reconstrução de conhecimentos, habilidades e atitudes. Nesse processo, há necessidade de reflexão sobre essa relação pedagógica preceptor-residente por parte dos preceptores para continuidade da qualificação dos atributos pedagógicos no exercício da preceptoria.

Este artigo trouxe uma proposta de um instrumento para avaliação pedagógica que se apresentou como formativa para a preceptoria em MFC no âmbito da APS do município do Rio de Janeiro, uma vez que promoveu espaços de discussão e reflexão sobre as práticas pedagógicas na preceptoria e a sensibilização para construção de planos de desenvolvimento dos atributos pedagógicos apresentados, considerando-se potencialidades e obstáculos no contexto em que esses preceptores estavam inseridos. 
Dessa forma, supõe-se que o instrumento produziu ressignificação para esses preceptores, um precursor de motivação e esperança na pretensão do desenvolvimento pedagógico desses profissionais. Espera-se que esse instrumento contribua para a proximidade aos ideais de educação libertadora e problematizadora na relação preceptor-residente, possibilitando a formação de profissionais com consciência crítica.

\section{Contribuição dos autores}

Concepção e delineamento do estudo: PRM, VFR.

Aquisição, análise ou interpretação dos dados: PRM, VFR.

Redação preliminar: PRM.

Revisão crítica da versão preliminar: VFR.

Todos os autores aprovaram a versão final e concordaram com prestar contas sobre todos os aspectos do trabalho.

\section{Conflitos de interesse}

PRM: Não há.

VFR: Não há.

\section{Agradecimentos}

Aos preceptores participantes desta pesquisa.

\section{REFERÊNCIAS}

1. Ministério da Saúde (BR). Biblioteca Virtual em Saúde [Internet]. Declaração de Alma-Ata sobre Cuidados Primários. Brasília (DF): Ministério da Saúde;2002 [Internet]; [acesso em 2018 Dez 4]. Disponível em: https://bvsms.saude.gov.br/bvs/publicacoes/declaracao_ alma_ata.pdf

2. Ministério da Saúde (BR). Secretaria de Atenção à Saúde. Departamento de Atenção Básica. Política Nacional de Atenção Básica. Brasília (DF): Ministério da Saúde; 2012 [Internet]; [acesso em 2020 Nov 22]. Disponível em: http://189.28.128.100/dab/docs/publicacoes/ geral/pnab.pdf

3. Ministério da Educação (BR). Secretaria de Educação Superior. Resolução CNRM no 1, de 25 de maio de 2015 . Regulamenta os requisitos mínimos dos programas de residência médica em Medicina Geral de Família e Comunidade - R1 e R2 e dá outras providências. Diário Oficial da União, Brasília (DF), 26 mai 2015 [Internet]; [acesso 2018 Nov 16]; Seção 1, 11-12. Disponível em: http://portal.mec. gov.br/index.php?option=com_docman\&view=download\&alias=20741-res01-25052015-cnrm-regulamenta-requisitos-pdf\&category_ slug=setembro-2015-pdf\&ltemid=30192

4. Secretaria Municipal de Saúde do Rio de Janeiro (SMS-RJ). Subsecretaria de Atenção Primária, Vigilância e Promoção da Saúde (SUBPAV) [Internet]. Cobertura da ESF. Rio de Janeiro (RJ): Prefeitura Municipal do Rio de Janeiro; 2020; [acesso em 2020 Abr 17]. Disponível em: <https://subpav.org/cnes/

5. Izecksohn MMV, Teixeira Junior JE, Stelet BP, Jantsch AG. Preceptoria em Medicina de Família e Comunidade: desafios e realizações em uma Atenção Primária à Saúde em construção. Ciência \& Saúde Coletiva. 2017; 22(3):737-746.

6. Melo EA, Mendonça MHM, Teixeira, M. A crise econômica e a atenção primária à saúde no SUS da cidade do Rio de Janeiro, Brasil. Ciência \& Saúde Coletiva. 2019; 24(12):4593-4598.

7. Mendes CLA. Perfil do profissional médico na Estratégia de Saúde da Família no município do Rio de Janeiro: um modelo em transição [Dissertação de Mestrado]. Rio de Janeiro (RJ): Escola Nacional de Saúde Pública Sergio Arouca; 2015.

8. Sociedade Brasileira de Medicina de Família e Comunidade (SBMFC) [Internet]. Currículo Baseado Em Competências Para Medicina de Família e Comunidade. Rio de Janeiro (RJ): SBMFC;2014; [acesso em 2018 Nov 11]. Disponível em: http://www.sbmfc.org.br/media/ Curriculo\%20Baseado\%20em\%20Competencias(1).pdf 
9. Castells MA. Estudo dos programas de residência médica em medicina de família e comunidade do Rio de Janeiro: a questão da preceptoria. [Dissertação de Mestrado]. Rio de Janeiro (RJ): Escola Nacional de Saúde Pública Sergio Arouca; 2014.

10. Botti SHO, Rego STA. Docente-clínico: O complexo papel do preceptor na residência médica. Physis: Revista de Saúde Coletiva. 2011; 21(1):65-85, 2011.

11. Villardi ML, Cyrino EG, Berbel NAN. Mudança de paradigma no ensino superior em saúde e as metodologias problematizadoras. In: Villardi ML, Cyrino EG, Berbel NAN, autoras. A problematização em educação em saúde: percepções dos professores tutores e alunos. São Paulo: Editora UNESP; 2015. p. 23-44.

12. Freire P. Pedagogia do oprimido. 67ª ed. Rio de Janeiro: Paz e Terra; 2019.

13. Hooks B. Ensinando a transgredir: a educação como prática da liberdade. $2^{\mathrm{a}}$ ed. São Paulo: Editora WMF Martins Fontes; 2017.

14. Perrenoud P. Construir as Competências desde a Escola. Porto Alegre: Artmed Editora; 1999.

15. Perrenoud P. Dez novas competências para ensinar. Porto Alegre: Artmed; 2000.

16. Depresbiteris L. Avaliação da Aprendizagem do Ponto de Vista Técnico-Científico e Filosófico-Político. In: Série Ideias n. 8. São Paulo: FDE; 1998. p.161-172.

17. Depresbiteris L. A avaliação na educação básica: ampliando a discussão. Estudos em Avaliação Educacional. 2001; (24):137-146.

18. Minayo MCS, Deslandes SF, Gomes R. Pesquisa Social: Teoria, método e criatividade. Petrópolis-RJ: Editora Vozes; 2016.

19. Kaufmann JC. A entrevista compreensiva: Um guia para pesquisa de campo. $3^{\underline{a}}$ ed. Petrópolis-RJ: Editora Vozes; 2013.

20. Haguette TMF. Metodologias Qualitativas na Sociologia. 14ª ed. Petrópolis-RJ: Editora Vozes; 2013.

21. Bardin L. Análise de conteúdo. São Paulo: Edições 70; 2016.

22. Associação Brasileira de Saúde Coletiva - Abrasco [Internet]. Notas Oficiais ABRASCO: A crise que esmaga a saúde do Rio de Janeiro. Rio de Janeiro(RJ): ABRASCO; 2018; [acesso em 2019 Nov 22]. Disponível em: https://www.abrasco.org.br/site/noticias/ posicionamentos-oficiais-abrasco/crivella-e-crise-que-esmaga-saude-do-rio-de-janeiro/38022 